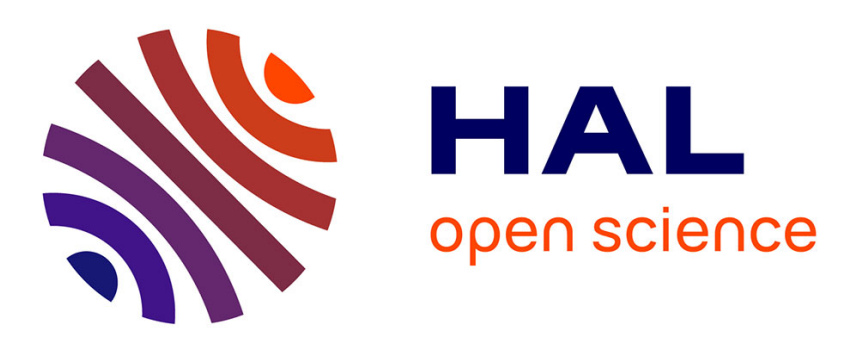

\title{
Impact of saturation on dispersion and mixing in porous media: Photobleaching pulse injection experiments and shear-enhanced mixing model
}

Joaquín Jiménez-Martínez, Tanguy Le Borgne, Hervé Tabuteau, Yves Méheust

\section{- To cite this version:}

Joaquín Jiménez-Martínez, Tanguy Le Borgne, Hervé Tabuteau, Yves Méheust. Impact of saturation on dispersion and mixing in porous media: Photobleaching pulse injection experiments and shear-enhanced mixing model. Water Resources Research, 2017, 53 (2), pp.1457-1472. 10.1002/2016WR019849 . insu-01471490

\section{HAL Id: insu-01471490 \\ https://hal-insu.archives-ouvertes.fr/insu-01471490}

Submitted on 20 Feb 2017

HAL is a multi-disciplinary open access archive for the deposit and dissemination of scientific research documents, whether they are published or not. The documents may come from teaching and research institutions in France or abroad, or from public or private research centers.
L'archive ouverte pluridisciplinaire $\mathbf{H A L}$, est destinée au dépôt et à la diffusion de documents scientifiques de niveau recherche, publiés ou non, émanant des établissements d'enseignement et de recherche français ou étrangers, des laboratoires publics ou privés. 


\section{Water Resources Research}

\section{RESEARCH ARTICLE \\ 10.1002/2016WR019849 \\ Key Points: \\ - Photobleaching experiments in two- \\ Impact of saturation on dispersion and mixing in porous media: Photobleaching pulse injection experiments and shear-enhanced mixing model} dimensional micromodels

- Ballistic dispersion at short-term as

soon as saturation is lowered

- Mixing is enhanced by fluid

deformation, stretching regime,

followed by a dissipation regime

Correspondence to:

J. Jiménez-Martínez,

joaquin.jimenez@eawag.ch;

jjimenez@ethz.ch; and

Y. Méheust,

yves.meheust@univ-rennes1.fr

Citation:

Jiménez-Martínez, J., T. Le Borgne,

H. Tabuteau, and Y. Méheust (2017),

Impact of saturation on dispersion and

mixing in porous media:

Photobleaching pulse injection

experiments and shear-enhanced

mixing model, Water Resour. Res., 53,

doi:10.1002/2016WR019849.

Received 27 SEP 2016

Accepted 19 JAN 2017

Accepted article online 24 JAN 2017

\author{
Joaquín Jiménez-Martínez1,2,3, Tanguy Le Borgne³, Hervé Tabuteau, and Yves Méheust³ \\ ${ }^{1}$ Department Water Resources and Drinking Water, EAWAG, Dubendorf, Switzerland, ${ }^{2}$ Department of Civil, Environmental \\ and Geomatic Engineering, ETH Zurich, Zurich, Switzerland, ${ }^{3}$ Geosciences Rennes, UMR 6118, CNRS, Université de Rennes, \\ Rennes, France, ${ }^{4}$ IPR, Université de Rennes 1-CNRS, UMR 6251, Campus Beaulieu, Rennes, France
}

\begin{abstract}
The dynamics of solute dispersion and mixing in unsaturated flows is analyzed from photobleaching experiments in two-dimensional porous micromodels. This technique allows producing pulse line (delta-Dirac) injections of a conservative tracer by bleaching a finite volume of fluorescent without disturbing the flow field. The temporal evolution of the concentration field and the spatial distribution of the air and water phases can be monitored at pore scale. We study the dispersion and mixing of a line of tracer under different water saturations. While dispersion in saturated porous media follows an approximately Fickian scaling, a shift to ballistic scaling is observed as soon as saturation is lowered. Hence, at the time scale of observation, dispersion in our unsaturated flows is dominated by the ballistic separation of tracer blobs within the water phase, between trapped clusters and preferential flow paths. While diffusion plays a minor role in the longitudinal dispersion during the time scale of the experiments, its interplay with fluid deformation is apparent in the dynamics of mixing. The scalar dissipation rates show an initial stretching regime, during which mixing is enhanced by fluid deformation, followed by a dissipation regime, during which diffusion overcomes compression induced by stretching. The transition between these two regimes occurs at the mixing time, when concentration gradients are maximum. We propose a predictive analytical model, based on shear-enhanced diffusion, that captures the dynamics of mixing from basic unsaturated porous media parameters, suggesting that this type of model may be a useful framework at larger scales.
\end{abstract}

\section{Introduction}

The transport of solutes in multiphase flows plays an important role in many environmental, including contaminant (bio-)remediation [Rolle et al., 2009; Williams et al., 2009], geological storage of $\mathrm{CO}_{2}$ [Orr, 2009] or of gases such as $\mathrm{H}_{2}$ [Panfilov, 2010], nuclear waste disposal [Winograd, 1981], as well as in industrial applications such as enhanced oil recovery [Liu et al., 2004; Jiménez-Martínez et al., 2016], geothermal energy [Barbier, 2002], or unconventional gas production [Middleton et al., 2015]. The most prominent environmental example is the vadose zone, which play a key role in the transport of chemical substances from the surface to groundwater resources. In particular, it acts as a buffer of pesticides and fertilizers (mainly nitrates) [Rockstrom, 2009; Sebilo et al., 2013], constrains the use of unconventional water for irrigation [Lahav et al., 2010], and plays a major role in controlling the contamination of primary groundwater bodies [Bolster et al., 2009], as well as artificial recharge operations [Bouwer, 2002; Sani et al., 2013].

In the unsaturated zone, where water and air coexist in the pore space, the spatial distribution of the two phases at pore scale evolves in a manner that is controlled by the average displacement velocity [Løvoll et al., 2004; Toussaint et al., 2005], the viscosity contrasts between the two fluids [Lenormand, 1990], and the stabilizing or destabilizing role of gravity [Méheust et al., 2002], as well as by the injection configuration [Tallakstad et al., 2009a; Toussaint et al., 2012]. This is also true for other types of biphasic flows, such as the flow of oil, water and gas in oil reservoirs. The spatial distribution of fluid phases controls the velocity field in the liquid phase. In general, a decrease in the water saturation is known to widen the velocity field distribution corresponds, as regions of fluid of very low velocities, including regions where it is trapped, coexist with connected preferential channels where velocities are high [de Gennes, 1983]. (c) 2017. American Geophysical Union All Rights Reserved. 
Solute transfers through the unsaturated zone are impacted accordingly. They are difficult to characterize and model since they depend on the water content (saturation), and more generally on the spatiotemporal distribution of the two phases (water and air), as well as on the variability in the water velocities. In this highly heterogeneous physical (i.e., coexistence of several phases: gas, liquid, and solids) and chemical context (e.g., spatial variability of redox conditions), (bio-)geochemical reactions can take place between the invading fluid and the resident one, or with the solids. In the latter case, the reactions may generate drastic changes in the physical (porosity, tortuosity) and hydraulic properties (permeability) of the medium.

The impact of saturation (water content) on solute dispersion and mixing is still matters of debate. Dispersion describes continuous increase in time of the spatial extent of a solute plume; it is mostly a consequence of the heterogeneity in the velocity field. An increase in the measured dispersion when the water saturation is decreased has been observed [e.g., Nutzmann et al., 2002; Maraqa et al., 1997; Haga et al., 1999; Padilla et al., 1999], but also the opposite behavior [e.g., Vanderborght and Vereecken, 2007; Birkholzer and Tsang, 1997]. Dispersion, in any case, does not directly inform on the spatial distribution of concentrations affecting chemical reactions. Mixing, on the other hand, is the process that controls those concentration fields by increasing the actual volume occupied by the solute and smoothing out concentration contrasts. In term of mixing, a generalized view is that in unsaturated flows preferential flow paths are more pronounced, which implies that travel times are shorter, or equivalently, that residence times allowing for solute mixing and potential chemical reactions are shorter [e.g., Vanderborght et al., 2001; Ursino et al., 2001; Persson et al., 2005; Gouet-Kaplan and Berkowitz, 2011; Kapetas et al., 2014]. However, this understanding is not always correct since flow channeling may also sustain concentration gradients transverse to the mean flow direction (i.e., from the edges of solute fingers), and consequently, enhance diffusive mass transfer and reaction rates [e.g., Jha et al., 2011; Jiménez-Martínez et al., 2015].

Quantitative experimental or numerical analysis of mixing need to resolve the mixing scale, which is the scale below which the concentration field can be considered uniform Le Borgne et al. [2011]. For transient processes, such as reactions coupled to solute transport, that scale is usually smaller than the pore scale. Therefore, pore scale investigations, as addressed in this work, are essential to understand solute transport by multiphase flows, and for the development of multiscale models allowing to predict reactive transport accurately. In Jiménez-Martínez et al. [2015], we presented a first set of pore scale experiments based on a two-dimensional (2-D) synthetic porous medium. Our investigation of solute mixing under continuous injection of tracer suggested a strong impact of water saturation on the mixing dynamics. Namely, the development of preferential channels upon decreasing water saturation was found to sustain concentration gradients in the direction perpendicular to the main flow direction, thus rendering the unsaturated flow more efficient at mixing the resident and injected liquids than a saturated flow. This crucial role of preferential channels was confirmed by a comparison of the measured mean concentration gradient to a simple estimate based on a conceptual relation between that mean concentration gradient, the mean concentration and the typical width of preferential channels. Later, Karadimitriou et al. [2016] studied the impact of saturation on dispersion using a similar type of experimental setup, although with a different porous geometry. Here we present an analysis of dispersion and mixing in a 2-D porous medium, based on a new experimental technique allowing for pulse Dirac injections with unprecedented precision in the geometric definition of the solute line and without any disturbance in the velocity field, which is generally very difficult to achieve experimentally. This injection condition is key to understand the spreading and mixing dynamics because it corresponds to the most common hypothesis under which models are derived and temporal scalings are defined. Although concentration fields for the Dirac injection conditions can be obtained in theory from differentiating concentration fields measured from the continuous injection conditions, in practice this comes at the cost of introducing significant experimental noise. Hence, from these new experimental observations, our objective is (i) to obtain a precise characterization of the impact of saturation on the spreading and mixing dynamics in porous media in terms of temporal scaling behavior under Dirac injection, and (ii) to derive a predictive analytical model for mixing in unsaturated porous media flow, which may be defined from basic physical parameters controlled by the geometry of the medium and by the physical properties of the fluids and solute tracer.

The manuscript is organized as follows. In section 2, we present the photobleaching technique that we have developed to perform controlled Dirac injections. In section 3, we discuss the main experimental observations of flow properties and of dispersion and mixing dynamics for different water saturations. In 
section 4, we derive a predictive analytical model quantifying the impact of saturation on mixing, and compare its predictions to the experimental measurements.

\section{Materials and Methods}

\subsection{Experimental Setup}

The experimental flow cell consists in a two-dimensional analogous porous medium in which the spatial distribution of the two fluid phases (wetting and nonwetting) and the concentration field in the liquid (wetting) phase can be measured precisely. This setup is complementary to that presented by Jiménez-Martínez et al. [2015], which allowed studying mixing processes in multiphase systems under continuous tracer injection.

A two-dimensional (2-D) porous medium (water-wet or hydrophilic) is used; it consists if a mono-layer of 4421 cylindrical grains of different diameters (Figure 1) sandwiched between two glass plates. The medium's dimensions along the plates are $L \times W=132 \times 87 \mathrm{~mm}$ while its thickness is $h=0.5 \mathrm{~mm}$. The mean grain diameter ( \pm standard deviation) is $d=0.83 \pm 0.22 \mathrm{~mm}$, the average pore throat $a=1.17 \mathrm{~mm}$, and the average pore size $\lambda=1.85 \mathrm{~mm}$. The porosity is $\phi=0.71$, the absolute permeability $\kappa=7.38 \times 10^{-3}$ $\mathrm{mm}^{2}$ (estimated from a modified Kozeny-Carman equation [Pinela et al., 2005] that considers cylinders instead of spheres), respectively. Note that this porosity is larger than those expected in real soils.

A $60-40 \%$ by weight water-glycerol solution, dyed with Fluorescein, is used as wetting phase; its viscosity is $\mu_{\mathrm{w}}$ $=3.72 \times 10^{-2} \mathrm{~kg} \mathrm{~m}^{-1} \mathrm{~s}^{-1}$, its density $\rho_{\mathrm{w}}=1.099 \times 10^{3} \mathrm{~kg} \mathrm{~m}^{-3}$. Air is used as the nonwetting fluid; its viscosity is $\mu_{\mathrm{nw}}=1.86 \times 10^{-5} \mathrm{~kg} \mathrm{~m}^{-1} \mathrm{~s}^{-1}$, its density $\rho_{\mathrm{nw}}=1.204 \mathrm{~kg} \mathrm{~m}^{-3}$. Consequently the viscosity ratio is $M=\mu_{\mathrm{nw}} / \mu_{\mathrm{w}} \sim 10^{-3}$, and the density ratio $H=\rho_{\mathrm{nw}} / \rho_{\mathrm{w}} \sim 10^{-3}$. The surface tension between these two fluids is $\gamma=6.914 \times 10^{-2} \mathrm{~N} \mathrm{~m}^{-1}$. The diffusion coefficient $D$ of the Fluorescein for this solution is $1.049 \times 10^{-4} \mathrm{~mm}^{2} \mathrm{~s}^{-1}$.

The optical acquisition setup is identical to that of Jiménez-Martínez et al. [2015]. In particular, we use optical pass band filters to ensure that only the light emitted by the fluorescein is recorded by the camera. A camera calibration (light intensity versus concentration) is carried out following de Anna et al. [2014a]. Although

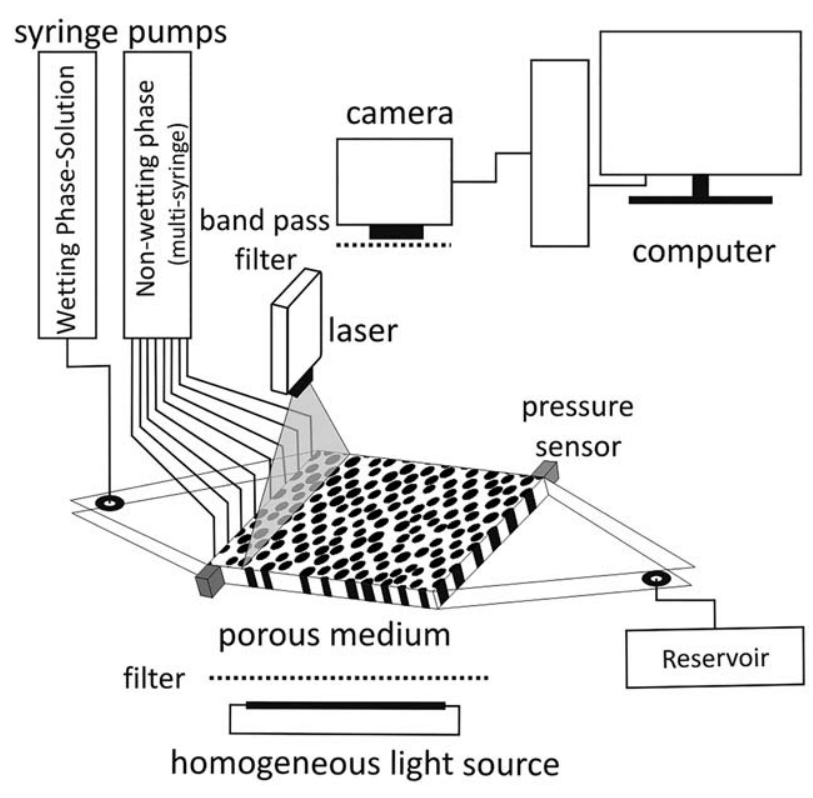

Figure 1. Scheme of the experimental setup. To perform partially saturated tests, the wetting (solution) and nonwetting (air) fluid are injected from the inlet line and pipes, respectively, and flow together for $1 \mathrm{~h}$, providing a homogeneous mixture of the two phases. Subsequently, a "dark" line is created in the solution by laser photobleaching. The line is displaced and deformed through the porous medium by further injection of the solution. A homogeneous enlightment excites the fluorescent tracer. A combination of optical filters allows to only capture the photons emitted by the fluorescent tracer. the dye is excited from below with a panel light source that produces a spatially homogeneous intensity, we normalize each raw image by an image of the porous medium saturated with the background solution at $C_{b}$, pixel by pixel, so as to ensure that the measurement of the concentration field of the tracer in the water phase is not impacted by small spatial heterogeneities of the light source. We thus measure the concentration field of the tracer with a resolution of $\simeq 0.05$ $\mathrm{mm}$ per pixel and a relative uncertainty on the measured concentration smaller than $0.2 \%$ de Anna et al. [2014a].

Two main experimental novelties have been introduced with respect to the setup used by Jiménez-Martínez et al. [2015]. The first one, and the most remarkable, is the use of a laser diode (LBX Oxxius) of wave length $494 \mathrm{~nm}$ and power $150 \mathrm{~mW}$ to create the initial pulse line injection by desactivating the Fluorescein molecules, a technique called photobleaching [Cuenca and Bodiguel, 2012]. The photobleaching of fluorescein is known to be irreversible [Imamura and Koizumi, 1955]. The second 
novelty is the monitoring of the global pressure drop $\Delta P_{L}$ along the medium, during the experiments. This is achieved by recording the pressure at two different positions (see Figure 1), respectively, close to the inlet and at a distance $L$ from the inlet along the mean flow direction, using SensorTechnics 26PC0070G6G Flow Trough pressure sensors. Over a cross section transverse to the mean flow direction, the pressure will only vary by small viscous and capillary fluctuations. Given the size of the porous medium and the viscosity of the wetting phase, the measured $\Delta P_{L}$ is much larger than those fluctuations [Tallakstad et al., 2009b]. Pressure measurements and photos of the porous medium are recorded at regular time intervals $(2 \mathrm{~s})$ after the start of the experiment (which is defined in the description of the experimental protocol, below).

\subsection{Experimental Protocol}

The porous medium is initially saturated with a solution of concentration $C_{\mathrm{b}}=350 \mathrm{mg} \mathrm{L}^{-1}$, which provides a strong contrast between the wetting and nonwetting phase. For the desaturation of the system, the nonwetting (air) and wetting (same solution) fluids are injected simultaneously, so that drainage and imbibition occur simultaneously in the medium (i.e., transient flow regime) [e.g., Tallakstad et al., 2009a]. The wetting saturation $S_{w}$ (defined from the image analysis as the ratio of the combined surface of wetting clusters to that of the porous space) is controlled from the ratio between the flow rates of the nonwetting $\left(Q_{n w}\right)$ and wetting $\left(Q_{w}\right)$ fluids. Once the pressure difference $\Delta P_{L}$ stabilizes, meaning that the system has reached a dynamic steady state flow regime (i.e., pressure difference, saturation, relative permeabilities, and cluster distribution fluctuate are statistically constant) [Tallakstad et al., 2009b], the fluid pair injection is stopped, resulting in a static mixture of the two phases.

Subsequently, a defined region (i.e., line) of the porous medium is exposed to a laser sheet (of width $1 \mathrm{~mm}$ ) at power $150 \mathrm{~mW}$ during $3600 \mathrm{~s}$, causing bleaching of the solution without disturbing the flow field. We thus create a "dark" line in the solution, perpendicular to the mean flow direction and of typical width $\sim 2 \mathrm{~mm}$. The duration of the bleaching procedure was adjusted to the best compromise between a good intensity contrast and a limited blurring of the line due to molecular diffusion during the bleaching procedure. Note that the Fluorescein is slightly photobleached around the main line due to transmission through the liquid of light scattered horizontally. This effect is more pronounced in unsaturated conditions due to the lensing-induced photobleaching, which is associated to the presence of gas-liquid interfaces [Sinton et al., 2003].

After completion of the photobleaching procedure, the background solution $C_{\mathrm{b}}$ is injected at the inlet at a constant flow rate $Q$, displacing the "dark" line through the porous medium, which causes the line to deform over time as it moves. Note that the choice of flow rate $Q$ is based on the criterium that all interfaces should remain locked in permanent positions, that is, that the subsequent flow of the aqueous phase does not perturb the established geometry. In what follows, we shall now consider the reduced concentration $c=1-\left(C / C_{b}\right)$, where $C$ is the measured concentration.

The flow and transport regimes are characterized by three dimensionless numbers. The Reynolds number, which estimates the ratio of the magnitude of inertial forces to that of viscous forces, is defined as $R e=\rho_{\mathrm{w}} \bar{v} a / \mu_{\mathrm{w}}$, where $\bar{v}$ is the average flow velocity. The capillary number quantifies the relative magnitude of viscous to capillary forces; it is expressed as $C a=\mu_{\mathrm{w}} Q \lambda^{2} /(\gamma \kappa A)$, where $Q$ is the flow rate and $A$ is the cross-sectional area in the direction normal to the average flow direction [e.g., Méheust et al., 2002]. The Péclet number is used to characterize the relative importance of advective and diffusive effects during transport. In our geometry, computing it as the ratio of the characteristic diffusion time over a pore throat, $t_{\mathrm{d}}=a^{2} /(2 D)$, to the characteristic advection time over a typical pore size $\lambda, t_{\mathrm{a}}=\lambda / \bar{v}$, we obtain $P e=\bar{v} a^{2} /(2 D \lambda)$.

The two experimental control parameters are the saturation $S_{w}$ and the imposed volumetric flow rate $Q$ (see Table 1). All investigated flow rates correspond to noninertial flows (Table 1), i.e., very low Reynolds numbers $(R e \ll 1)$, typical in porous media, which corresponds to laminar flows (so-called Stokes flows), so that in effect the flow process is characterized by the saturation and by the capillary number. Note however that despite the fact the geometry of the porous medium is invariant along the vertical, the flow velocity field varies vertically due to the no-flow conditions at the top and bottom flow boundaries. The vertical velocity profile at a given horizontal position is controlled by the distance between the two nearest solid grains. In our medium that distance is usually of the same order as the medium thickness, so that the vertical velocity profile is neither parabolic, as it would if no solid grains were present between the horizontal 
Table 1. Control Parameters $S_{\mathrm{w}}$ (Saturation Degree) and $Q$ (Volumetric Flow Rate) for the Experiments, and Corresponding Mean Interstitial Flow Velocity $\bar{v}$, Reynolds $(R e)$, Péclet $(P e)$, and Capillary $(C a)$ Numbers

\begin{tabular}{lccccc}
$S_{w}$ & $Q\left(\mathrm{~mm}^{3} \mathrm{~s}^{-1}\right)$ & $\bar{v}\left(\mathrm{~mm} \mathrm{~s}^{-1}\right)$ & $\operatorname{Re}(-)$ & $P e(-)$ & \\
\hline 1.0 & 1.375 & 0.0440 & $1.52 \times 10^{-3}$ & 155 & \\
0.83 & 0.277 & 0.0107 & $3.72 \times 10^{-4}$ & 38 & $2.68 \times 10^{-5}$ \\
0.77 & 0.277 & 0.0115 & $3.98 \times 10^{-4}$ & 41 & $2.87 \times 10^{-5}$ \\
0.71 & 0.277 & 0.0124 & $4.31 \times 10^{-4}$ & 44 & $3.11 \times 10^{-5}$ \\
\hline
\end{tabular}

plates, nor a plug flow profile with narrow limit boudaries at the top and bottom boundaries. We denote it as quasi-2D.

\section{Experimental Observation of Flow, Dispersion, and Mixing in Unsaturated Media}

The experimental setup proposed above allows for the first time to analyze transport processes in unsaturated porous media under Dirac injection conditions. This is an important improvement over previous continuous injection protocols [Jiménez-Martínez et al., 2015; Karadimitriou et al., 2016] in two key aspects. First, the Dirac injection condition allows identifying the dispersion regimes directly through the temporal scaling of the second spatial moment of the plume, which is not possible for a continuous injection. This is particularly important for understanding the nature of the non-Fickian transport behaviors observed under unsaturated conditions. Secondly, the Dirac injection condition is ideal for understanding the dynamics of mixing as it allows observing the evolution of a single line of tagged tracer under the combined action of diffusion and the deformation due to velocity gradients. As discussed in the following, this injection condition allows identifying precisely the regimes over which one of these two mechanisms dominates over the other, and subsequently deriving an analytical mixing model.

Snapshots of the concentration field at different times, both under fully and partially saturated conditions, are shown in Figure 2. They illustrate the transport and deformation of the line of solute through the porous medium. The partially saturated experiments were performed at the same injection flow rate $\left(Q=0.277 \mathrm{~mm}^{3} \mathrm{~s}^{-1}\right)$. The saturation, capillary number and Péclet number involved are shown in Table 1.

\subsection{Flow Characterization: Cluster Size Distribution}

The pressure difference recorded between the inlet and outlet sensor, $\Delta P_{L}$, is measured under conditions of static mixture of the two phases and at constant flow rate $Q$, during the transport experiments. In the inset of Figure $3 a, \Delta P_{L}$ is plotted as a function of time and for different saturations. For all experiments, the pressure difference between the inlet and the outlet increases as saturation decreases.

A generalized Darcy law equation $\left(k_{r}=q\left(\mu_{\mathrm{w}} / k\right) / \nabla P\right.$, where $q$ is the Darcy velocity and $\left.\nabla P=\left|\Delta P_{L} / L\right|\right)$ is used to compute the wetting phase permeability value as a function of the saturation $S_{w}$ (Figure $3 a$ ). As commonly observed [e.g., Brooks and Corey, 1964], a power law function in the form $k_{r}\left(S_{w}\right)=0.942 \cdot S_{w}^{10.33}$ can be fitted to the data. Note that the large exponent results from the 2-D character of the experiments. A decrease in hydraulic conductivity occurs as water saturation decreases. This is expected as the pressure drop over the medium is built through the liquid (wetting) phase, whose viscosity is 1000 times that of the air. For a given imposed flow rate, replacing the liquid phase with air in some of the pores can only decrease the global pressure drop, and, therefore, the hydraulic conductivity. In addition, capillary forces make the displacement of water by air easier in larger pores (or pores with larger entry throats), so such larger pores are more easily occupied by the air. When the saturation is close to 1 , since larger pores bring the largest contribution to $\Delta P_{L}$ when they are filled by the liquid, the conductivity of the wetting phase decreases substantially as the fraction of connected larger saturated pores is reduced. Further decrease in the wetting phase saturation will imply the desaturation of smaller pores, whose contribution to $\Delta P_{L}$ when saturated is not as large. Hence, as the global saturation is decreased further, the decrease in the relative permeability $k_{r}$ becomes slower, as described by the power law behavior.

Images of the phases structure have been analyzed in order to determine the size distribution of nonwetting clusters (air bubbles). This distribution is one of the main controls on transport processes (i.e., dispersion and mixing). The probability density function for the gyration radius of air bubbles, $f(R)$, is shown in 
a. $\mathrm{S}_{\mathrm{w}}=1 \quad \mathrm{Pe}=155$
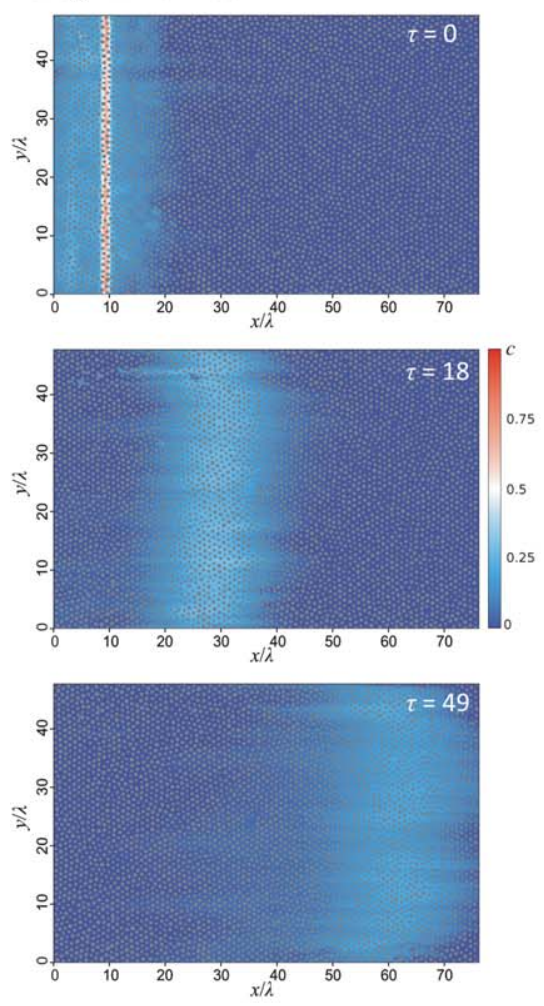

c. $\mathrm{S}_{\mathrm{w}}=0.77 \mathrm{Pe}=41$
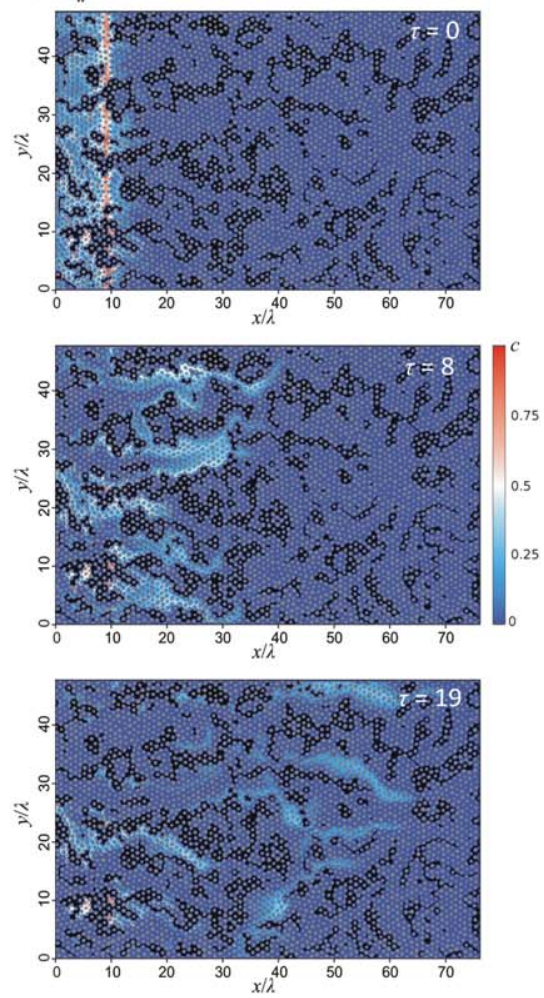

b. $\mathrm{S}_{\mathrm{w}}=0.83 \mathrm{Pe}=38$
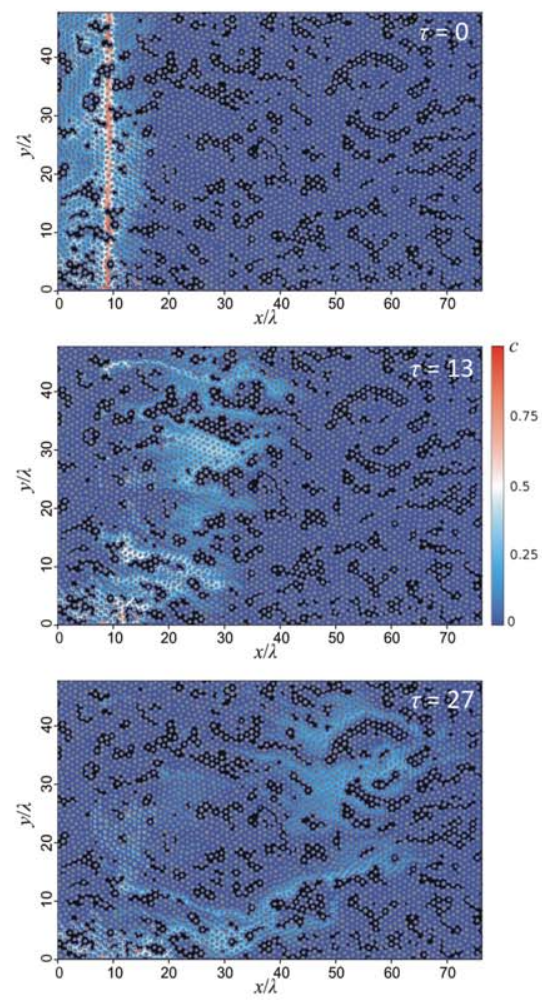

d. $S_{w}=0.71 P e=44$
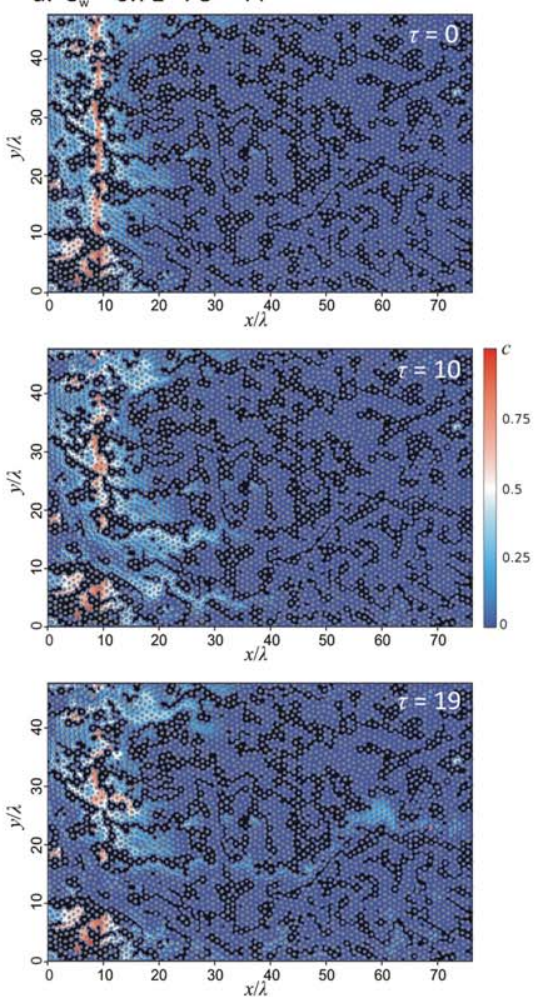

Figure 2. Spatial distribution of the normalized concentration $c(\boldsymbol{x}, \tau)$ obtained in fully (a) and partially (b-d) saturated conditions at different dimensionless times, $\tau=t / t_{\mathrm{a}}$ (air: black; grains: gray). In unsaturated conditions, preferential paths coexist with stagnation zones, where the tracer is diffused. 
a.

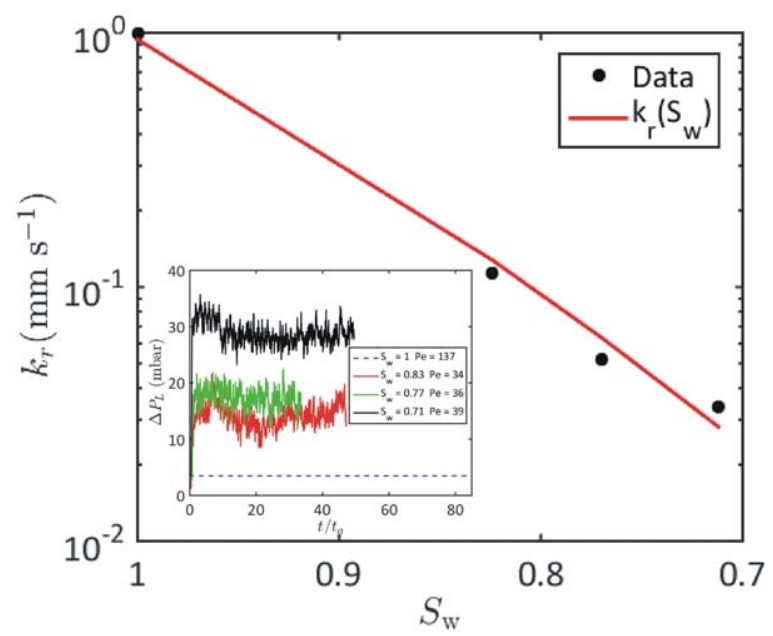

b.

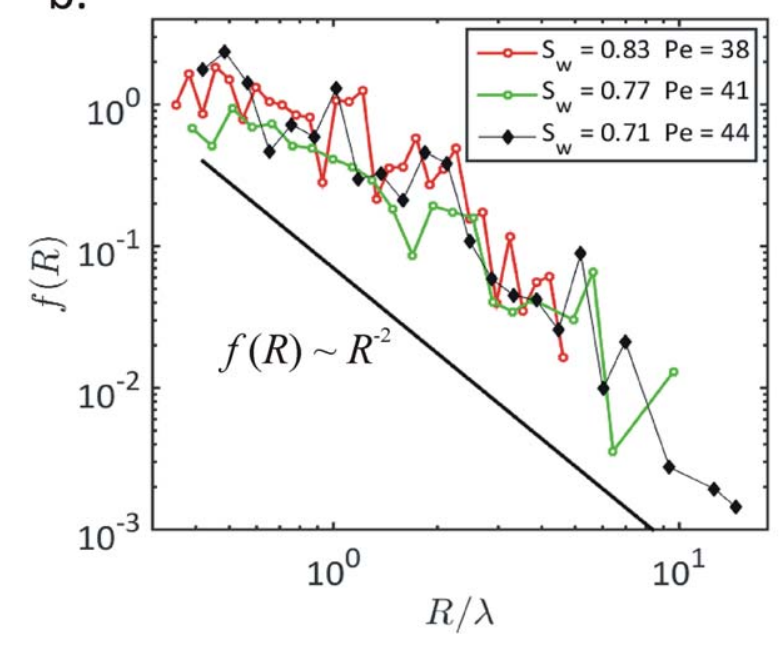

Figure 3. (a) Relative permeability function $\left(k_{r}\right.$ versus $\left.S_{w}\right)$ with the fitted power law model added. The inset shows the pressure drop over the model lengths, $\Delta P_{L}$, recorded as a function of time during the transport experiments; the spatial distribution of the phases is permanent. (b) Probability density function (PDF) $f(R)$ for the sizes of nonwetting (air) clusters, for the three investigated $S_{w}$. The cluster size is measured as a gyration radius $\sqrt{\mathcal{A} / \pi}$ from the area $\mathcal{A}$ of the cluster, and expressed in pore size units.
Figure $3 b$ as a function of $R / \lambda$, for all investigated saturations. The behavior is close to a power law with an exponent -2 (shown on the plot for visual comparison), in good agreement with Tallakstad et al. [2009b]'s findings on a similar system, but here the behavior is observed on a limited range of scales (1-2 decades), so that an exponential cutoff is clearly visible for cluster sizes approaching $\simeq 5$ times the typical pore size. The noise in the data could be reduced by averaging over a large number of equivalent configurations, but the range of scales verifying the power law behavior would still be limited. The desaturation (i.e., increase in the nonwetting phase content) gives rise to a broader distribution of air bubble sizes, associated with a lower hydraulic conductance of the aqueous phase. At sufficiently low saturations, transport in such systems may be considered as a percolation problem [Stauffer and Aharony, 1994; Rhodes and Blunt, 2007; Tallakstad et al., 2009b; Sahimi, 2012].

\subsection{Spreading}

A decrease in the saturation of the aqueous phase generally leads to a higher degree of heterogeneity of the fluid velocities in that phase, which impacts the associated transport phenomena [e.g., Le Borgne et al., 2008, 2010; Jiménez-Martínez et al., 2015]. Regions of fluid of low velocities, including regions of trapped fluid, coexist with high velocity regions (preferential paths) [de Gennes, 1983]. A visualization of the impact of the wetting phase spatial organization on solute transport is given by the longitudinal concentration profiles (Figure 4), defined as

$$
\bar{c}(x, t)=\int c(x, y, t) d y
$$

Under partially saturated conditions, and for the same dimensionless time $\left(\tau=t / t_{\mathrm{a}}\right)$, the longitudinal concentration distribution becomes strongly asymmetric in time with earlier breakthrough and much longer tailing than their saturated counterpart.

Since the experiments are performed under Dirac injection conditions, we can characterize the spreading dynamics directly from the time evolution of a characteristic plume size, $\sigma_{x}$. The temporal scaling of $\sigma_{x}$ allows understanding dispersion mechanisms without the need of fitting an a priori model to tracer breakthrough curves [Bouchaud and Georges, 1990]. One can distinguish in particular the Fickian scaling, $\sigma_{x} \sim t^{1 / 2}$, characteristic of uncorrelated random velocities, and the ballistic scaling $\sigma_{x} \sim t$, arising from perfectly correlated velocities with negligible diffusive mass exchange. A large spectrum of non-Fickian transport behavior can also be expected depending on the assumptions that can be made on the velocity distribution and correlation properties [Berkowitz et al., 2006; Benson et al., 2000]. 

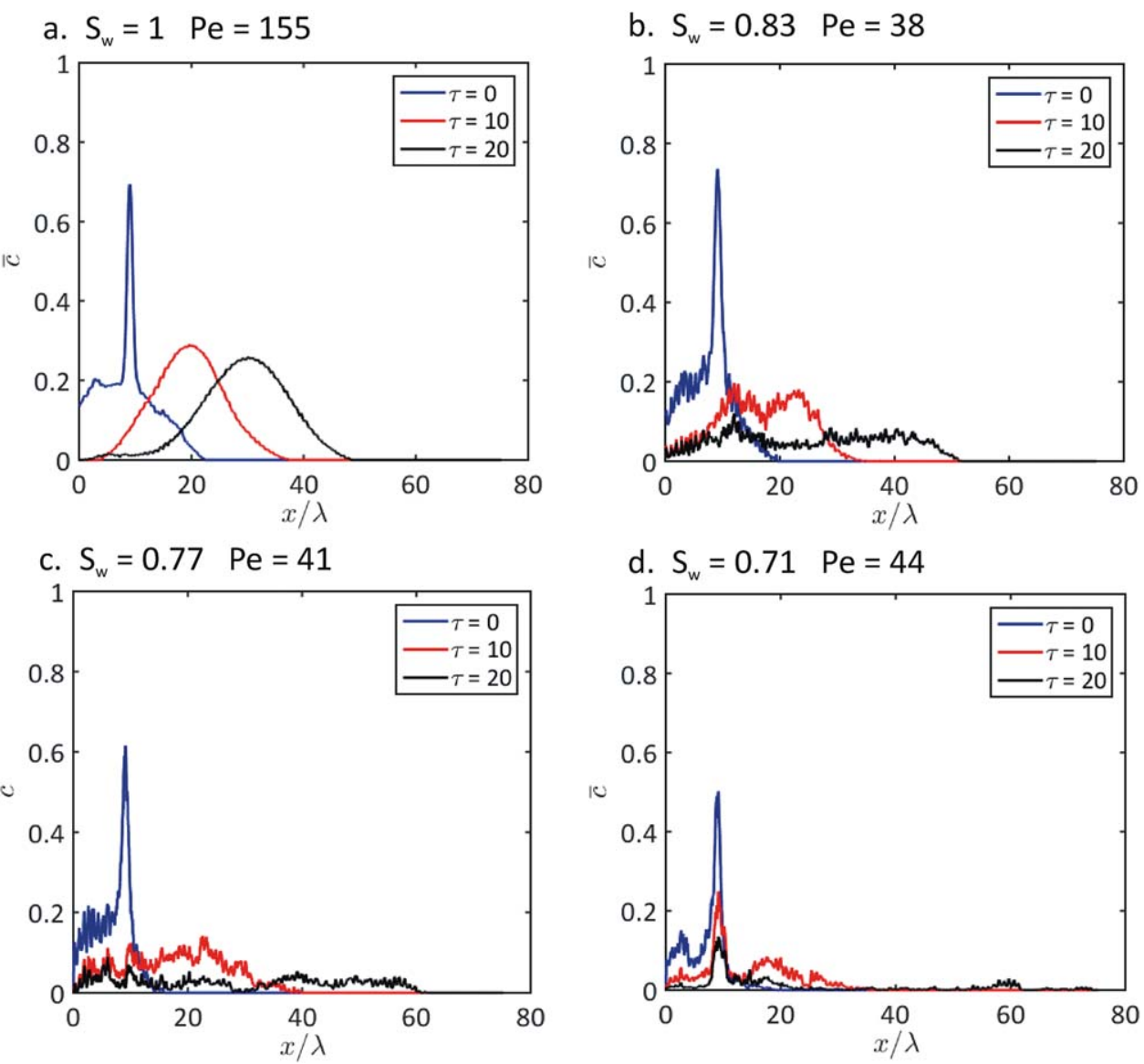

Figure 4. Longitudinal concentration profiles $\bar{c}(x, t)$, obtained in fully (a) and partially saturated (b-d) conditions at fixed times $\left(\tau=t / t_{\mathrm{a}}=0,10\right.$, and 20$)$.

We define as the longitudinal standard deviation of the concentration field $c$ :

$$
\sigma_{x}^{2}(t)=\frac{L_{y}}{M} \int d x[x-\langle x(t)\rangle]^{2} \bar{c}(x, t)
$$

where $M=L_{y} \int \bar{c}(x, t) d x$ is the total solute mass inside the system of width $L_{y}$, and $\langle x(t)\rangle=\left(L_{y} / M\right) \int x \bar{c}(x, t) d$ $x$ is the longitudinal position of the center of mass of the solute plume.

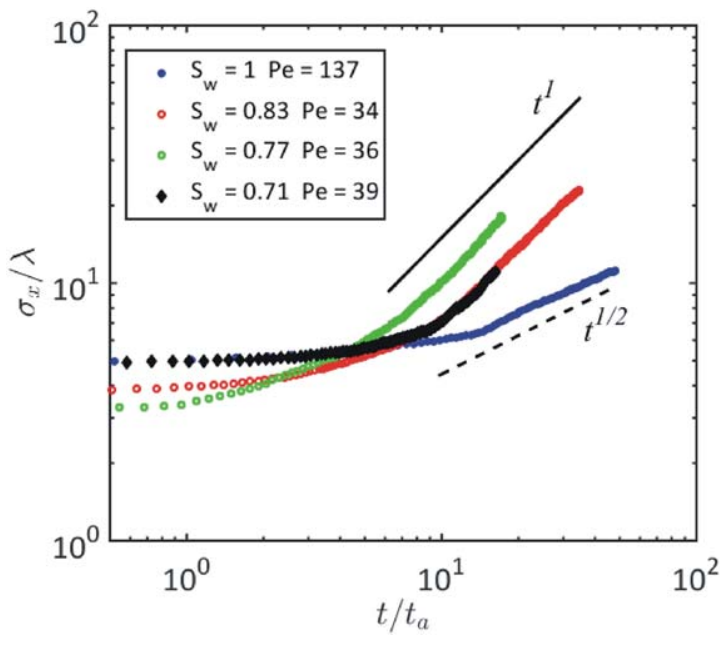

Figure 5. Characteristic normalized spreading length $\sigma_{x}(t)$ (longitudinal standard deviation of the plume) for the four investigated saturations.
The temporal evolution of the characteristic plume size $\sigma_{x}(t)$ is shown in Figure 5 for different saturations. Note that the initial high value of $\sigma_{x}(t)$ in all experiments results from the slight blurring of the main bleaching line that occurs during the photobleaching procedure (see section 2.2). For the saturated case, we observe a Fickian behavior $\sigma_{x}(t) \sim t^{1 / 2}$ at sufficiently large times. However, for all unsaturated experiments we observe a super-diffusive spreading akin to a ballistic behavior $\sigma_{x}(t) \sim t$ [Bouchaud and Georges, 1990]. This behavior is associated to an organization of the solute cloud in welldeveloped solute fingers (Figure 2) as a consequence of the broad distribution of velocities associated to the highly channelized flow. The ballistic scaling suggests that diffusion does not play a measurable role on dispersion during the 


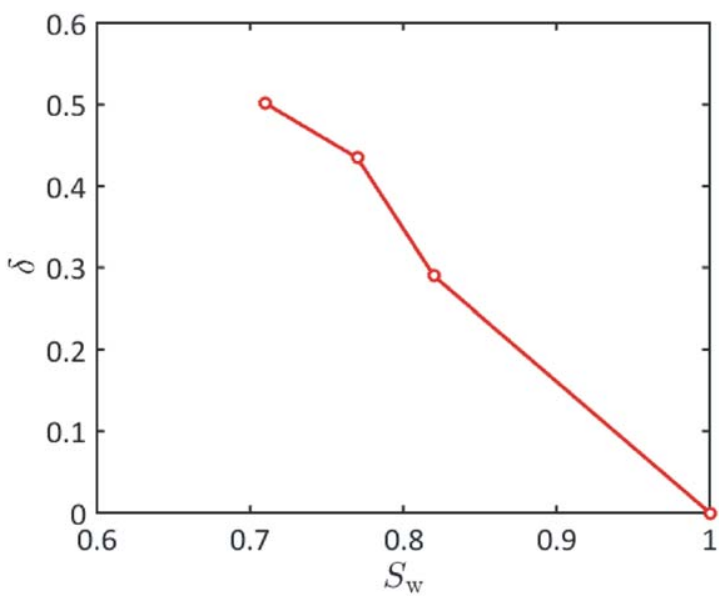

Figure 6. Fraction $\delta$ of the wetting phase not involved in the transport process, plotted as a function of $S_{w}$. time scale of the experiments. However, as discussed in the following section, diffusion is clearly significant for these experiments and it plays a major role for mixing. But, considering the strong flow channeling apparent in unsaturated flow experiments, it is likely that dispersion is dominated by the ballistic separation of trapped solute blobs and solute parcels traveling in high velocity channels. While the impact of molecular diffusion on the spreading remains limited in this regime, we expect that it will ultimately strongly affect spreading through diffusive mass transfer between low velocity zones and high velocity channels. Such an effect would be visible at larger time scales and over spatial scales larger than the size of the largest cluster in the system.

The impact on solute transport of the regions of very low water velocities that exist in unsaturated conditions, including regions where the solute is trapped, has been evaluated by computing the volume fraction $\delta$ of the aqueous phase that is not involved in the transport process. It was defined over the total duration of the experiments, that is, from the area not reached by the tracer at the end of the experiment: $\{(x, y) \mid 0 \leq c(x, y)<0.01\}$. Figure 6 shows how $\delta$ varies with $S_{w}$. The lower the wetting saturation, the larger the area of low velocity zones, which implies the focusing of the remaining flow onto narrower connected preferential channels of high velocity, resulting in a broader distribution of velocities. Therefore, desaturation enhances fingering and diffusion from the edges of the fingers to the low velocity zones, that is, diffusion mostly along a direction perpendicular to the mean flow direction.

\subsection{Mixing Dynamics}

The concentration fields obtained after a Dirac injection (Figure 2) provide new insights on the dynamics of mixing and its dependence on the saturation level. Previous experimental observations obtained under continuous injection conditions [Jiménez-Martínez et al., 2015] indicated the role played by the development of solute fingers along preferential flow channels in the enhancement of mixing in unsaturated porous media flows. From the Dirac injection experiments presented here, we can analyze in much more details the respective roles of fluid stretching and diffusion in controlling the mixing dynamics. These observations are key to the development of the predictive analytical model presented in the next section.

The global mixing rate is quantified from the scalar dissipation rate [e.g., Ottino, 1989; Le Borgne et al., 2010],

$$
\chi(t)=\int D\|\nabla c\|^{2} d x d y
$$

where || || denotes the vector magnitude. The interest of the scalar dissipation rate as a measure of mixing is that it is directly related to the global reaction for a fast reversible bimolecular reaction [e.g., de Simoni et al., 2005]. One of the main difficulties in evaluating the scalar dissipation rate and reaction rate is the estimation of the concentration gradients in equation (3). The concentration field can vary sharply over small distances, particularly for highly heterogeneous velocity fields as in unsaturated conditions. For a Dirac delta injection, the scalar dissipation rate $\chi(t)$ can be evaluated alternatively from the temporal derivative of the integral of the squared concentration over the system [e.g., Pope, 2000; Le Borgne et al., 2010]. If $\Omega$ denotes the mixing zone, which we define from the experimental images as $\Omega=\{(x, y) \mid c(x, y)>0.025\}$, and $\Sigma_{m}(t)$ is the area of $\Omega$, that is the characteristic area occupied by the solute, $\chi(t)$ can thus be evaluated as

$$
\bar{\chi}_{\Omega}(t)=-\frac{1}{2 \Sigma_{\mathrm{m}}(t)} \frac{d \Gamma_{\Omega}}{d t}
$$




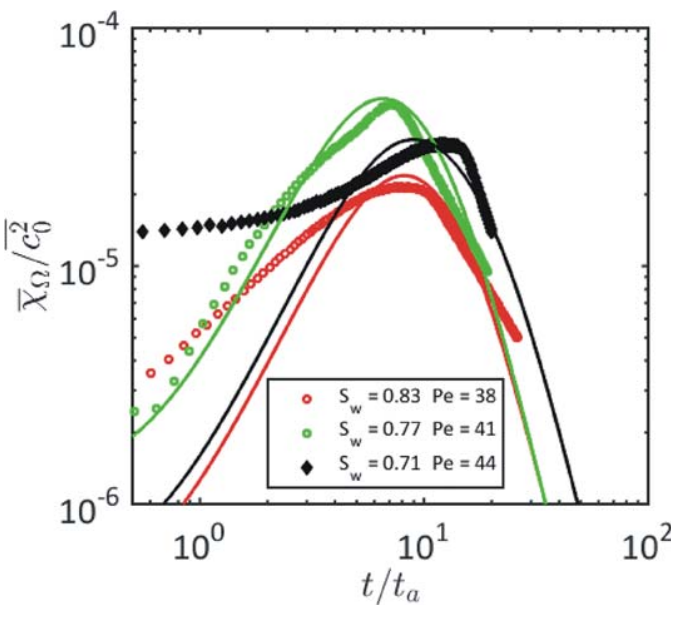

Figure 7. Scalar dissipation rate $\bar{\chi}_{\Omega}(t)$ measured as a function of time during the unsaturated experiments (symbols). For each of the investigated saturations, a solid line represents the scalar dissipation rate estimated analytically from the shear deformation model (equation (13))

$$
\text { with } \Gamma_{\Omega}(t)=\int_{\Omega} c^{2} d x d y
$$

This approach, as well as the lower threshold to define the mixing zone, are chosen in order to reduce possible sources of experimental noise. Note that $\Gamma_{\Omega}(t)$ quantifies the temporal variability of the concentration values, not the spatial extent of the plume. $\Gamma_{\Omega}(t)$ characterizes a mixing state in the sense that the better mixed the system is, the smaller $\Gamma_{\Omega}(t)$ is.

The evolution in time of the scalar dissipation rate reflects the efficiency of the mixing process (Figure 7). The finger structure developed in the unsaturated cases (Figure 2) as a consequence of the heterogeneous advecting velocity field, leads to a significant increase in the surface available for fluid mixing [e.g., Jha et al., 2011; Jiménez-Martínez et al., 2015], along which the concentration gradient develops mainly in the direction transverse to the average flow. The strong deformation of the mixing interface, i.e., in this study, of the solute line, and the consequent enhancement of mixing, are analogous to observations made in saturated highly heterogeneous porous media [e.g., Werth et al., 2006] and in Darcy scale heterogeneous flow topologies [de Barros et al., 2012; Le Borgne et al., 2014].

In the temporal evolution of $\bar{\chi}_{\Omega}(t)$ shown in Figure 7 for the unsaturated experiments, two main regimes are visible. In the first regime, an increase in the scalar dissipation rate, and therefore in the mixing rate, is observed. The mean concentration gradient $\overline{\nabla c}$ reaches a maximum at a characteristic $t_{\mathrm{s}}$, after which $\bar{\chi}_{\Omega}$ decreases in time. A similar mixing behavior has been observed for heterogeneous Darcy fields [de Dreuzy et al., 2012]. For the two largest saturations, a decrease in $t_{\mathrm{s}}$ and an increase in $\overline{\nabla c}\left(t_{\mathrm{s}}\right)$ is observed as saturation decreases. However, for the smallest water content $\left(S_{w}=0.71\right)$, the trend is inverted, i.e., $t_{\mathrm{s}}$ is higher and $\overline{\nabla c}\left(t_{\mathrm{s}}\right)$ is in between the values observed for the two other saturations.

The observed mixing behaviors differ from our previous observations made under continuous injection conditions, in which a slow monotonic decrease of the scalar dissipation rate was reported for unsaturated flows [Jiménez-Martínez et al., 2015]. This suggests that the injection condition has an important impact on the mixing dynamics. Under continuous injection conditions new concentration gradients can be continuously created by the slow increase of the concentration in preferential channels. Under pulse injection, the concentration gradients first increase sharply as the tracer line is stretched by the action of velocity gradients (see Figure 2), and then decay as diffusion takes over (Figure 7). This injection condition thus allows investigation more finely the coupling between flow heterogeneity, diffusion, and mixing in unsaturated flows. We explain and quantify these new observations in the following through the development of a predictive mixing model.

\section{Shear-Enhanced Mixing Model}

Since there is no new injection of solute mass in the domain after the pulse injection, an oversimplistic understanding would have one expect concentration gradients to decay continuously in time as diffusion and dispersion smooth out concentration differences. The observations of Figure 7 suggest that there is another mechanism for enhancing concentration gradients than that resulting from the continuous injection of solute mass, and that this new mechanism is dominant during an initial phase (for $t<t_{c}$ ). Here we propose to explain these observations by invoking the shear action of local velocity gradients, which is stretching the initial line of tracer through the domain (Figure 2). Since the flow can be considered incompressible, the elongation of the tracer line implies its simultaneous compression in the direction perpendicular to the main direction of elongation. This may counteract the action of diffusion, thus enhancing concentration gradients, at least for some time. 
Based on these experimental observations, we propose a mixing model to quantify the link between the dynamics of mixing and the properties of the advective velocity field. For this, we adapt the theoretical framework developed by Le Borgne et al. [2013, 2015], based on stretching-enhanced diffusion to the context of multiphase flow. This framework, developed and validated for saturated flows at the Darcy scale in heterogeneous permeability fields, quantifies the enhancement of diffusive mass transfer across a line of tracer by its elongation through velocity gradients. This mechanism is also expected to be active at pore scale through velocity gradients that develop between the center of pores and the walls, and between different flow paths [de Anna et al., 2014b]. As illustrated in Figure 2, the presence of two phases further increases the velocity differences across the domain, thus further enhancing mixing rates.

In two-dimensions, shear deformation, i.e., deformation due to gradients in longitudinal velocities along the direction transverse to streamlines, is expected to be the dominant deformation process [Le Borgne et al., 2015]. Here we consider a simple model of shear deformation based on a mean velocity gradient $\overline{\nabla v}$, leading to a linear elongation rate,

$$
\frac{L(t)}{L_{0}}=\sqrt{1+\overline{\nabla v}^{2} t^{2}}
$$

where $L(t)$ is the length of the tracer line, of initial length $L_{0}$. In incompressible flows, stretching implies compression in the direction perpendicular to the main elongation direction Meunier and Villermaux [2010]. As compression decreases the distance between concentration isolines, it is expected to increase concentration gradients. This effect, together with the increase in the area available for diffusive mass transfer, leads subsequently to stretching enhanced diffusion. This can be quantified as follows.

The average concentration gradient along the plume can be expressed as

$$
\overline{\nabla c}(t)=\frac{c_{\mathrm{m}}(t)}{\bar{s}(t)}
$$

where $c_{m}(t)$ is the average concentration in the tracer line and $\bar{s}(t)$ is the average local width of the line. Considering a strip of tracer, of initial length $L_{0}$ and initial width $s_{0}$, elongated by the flow in one direction and compressed in the other direction, concentration gradients are mainly oriented in the direction perpendicular to the direction of elongation. The evolution of a concentration profile along this direction can be expressed as a compression-diffusion equation Ranz [1979], Meunier and Villermaux [2010], and Le Borgne et al. [2015],

$$
\frac{\partial c}{\partial t}=\frac{1}{L} \frac{d L}{d t} \zeta \frac{\partial c}{\partial \zeta}+D_{\text {eff }} \frac{\partial^{2} c}{\partial \zeta^{2}}
$$

where $D_{\text {eff }}$ is the effective diffusion coefficient, $\zeta$ is the coordinate in the direction perpendicular to the elongation direction, and $(1 / L) /(d L / d t)$ is the compression rate induced by the elongation of the strip of length $L(t)$. Hence, the first term on the right hand side quantifies compression in the direction perpendicular to the direction of elongation. The solution of this equation is a Gaussian distribution with maximum [Ranz, 1979; Villermaux, 2012; Le Borgne et al., 2015]

$$
c_{\mathrm{m}}(t)=\frac{c_{0}}{\sqrt{1+4 \tau_{\mathrm{e}}(t)}}
$$

where $\tau_{\mathrm{e}}$ is the warped time, defined as

$$
\tau_{\mathrm{e}}(t)=\frac{D_{\text {eff }}}{s_{0}^{2}} \int_{0}^{t} d t\left(\frac{L(t)}{L_{0}}\right)^{2} .
$$

$\tau_{\mathrm{e}}$ quantifies the enhancement of diffusion by fluid stretching. In the absence of fluid stretching, $\overline{\nabla v}=0$ and $L(t) / L_{0}=1, \tau_{\mathrm{e}}(t)=\left(D_{\text {eff }} / s_{0}^{2}\right) t$ and the classical decay $c_{\mathrm{m}}(t) \sim s_{0} / \sqrt{D_{\text {eff }} t}$ is recovered. For linear shear, $L(t)$ is given by equation (6), and hence the warped time is $\tau_{\mathrm{e}}(t)=\left(D_{\text {eff }} / s_{0}^{2}\right)\left[t+\left(\overline{\nabla v}^{2} t^{3}\right) / 3\right]$. Consequently the decay of the maximum concentration is asymptotically faster, in the form $c_{\mathrm{m}}(t) \propto s_{0} /\left(\overline{\nabla v} \sqrt{D_{\text {eff }} t^{3}}\right)$, which is a consequence of stretching-enhanced diffusion. 
The temporal evolution of the average mixing scale $\bar{s}(t)$ is driven by the balance between compression perpendicular to the direction of elongation, which tends to reduce it, and molecular diffusion, which tends to increase it [Le Borgne et al., 2011; Villermaux, 2012]. $\bar{s}(t)$ can be obtained from the second spatial moment of the transverse concentration profile, solution of equation (8). For a linear shear, this leads to the following approximate evolution equation [Le Borgne et al., 2015],

$$
\bar{s}(t) \approx \sqrt{\frac{s_{0}^{2}}{1+\overline{\nabla v}^{2} t^{2}}+2 D_{\mathrm{eff}} t} .
$$

At initial times, compression dominates over diffusion and the evolution of the average lamella width is $\bar{s} \approx s_{0} /\left(1+\overline{\nabla v}^{2} t^{2}\right)^{1 / 2}$, while at later times, diffusion is expected to overcome compression and the diffusive scaling is recovered: $\bar{s}(t) \approx \sqrt{2 D_{\text {eff }} t}$. The crossover time between these two regimes is the mixing time [Villermaux, 2012; Le Borgne et al., 2015],

$$
t_{\mathrm{s}}=\left(\frac{s_{0}^{2}}{D_{\mathrm{eff}} \overline{\nabla v}^{2}}\right)^{1 / 3} .
$$

The temporal evolution of the average concentration gradient is obtained by inserting equations (9) and (11) in equation (7). The average scalar dissipation rate is related to the average concentration gradient by the relation $\bar{\chi}_{\Omega}(t)=D\left(\overline{\nabla c}^{2}(t)+\sigma_{\nabla c}^{2}(t)\right)$, where $\sigma_{\nabla c}^{2}=\overline{(\nabla c)^{2}}-(\overline{\nabla c})^{2}$. As $\sigma_{\nabla c}^{2}$ is found to be much smaller than $\overline{\nabla c}^{2}$, we use the simple approximation $\bar{\chi}_{\Omega}(t) \approx D \overline{\nabla c}^{2}(t)$ [e.g., Jiménez-Martínez et al., 2015]. Hence, we obtain an estimation of the global average scalar dissipation rate as

$$
\bar{\chi}_{\Omega}(t)=\frac{D}{\frac{s_{0}^{2}}{1+\overline{\nabla v}^{2} t^{2}}+2 D_{\mathrm{eff}} t} \frac{c_{0}^{2}}{1+4 \tau_{\mathrm{e}}(t)}
$$

This model is determined by three parameters, $s_{0}, D_{\text {eff, }}$ and $\overline{\nabla v}$, which can be estimated from basic physical principles as follows: $s_{0}$ is estimated as the width of the initial concentration profile; $D_{\text {eff }}$ is considered to be the Taylor-Aris or planar dispersion coefficient defined with the flow cell thickness as a relevant length scale, and being therefore proportional to the Péclet squared (and, consequently, to the mean velocity squared) [e.g., Bouquain et al., 2011]; and $\overline{\nabla v}$ is approximated as $\bar{v} / a$. The values corresponding to the different saturation degrees are summarized in Table 2 , and the corresponding time evolution of the estimated $\bar{\chi}_{\Omega} / c_{0}^{2}$ are plotted on top of the corresponding experimental data in Figure 7 . Based on these estimations of the three parameters, and without further fitting, equation (13) provides a relatively good estimate of the temporal evolution of the global average scalar dissipation rate in unsaturated conditions over the range of investigated times. In particular, this model represents well the initial increase in the mean concentration gradient (and therefore, in the scalar dissipation rate) due to the compression induced by fluid stretching, the balance of diffusion and compression at the mixing time $t_{\mathrm{s}}$, and the subsequent decay of concentration gradients. Note that in general equation (13) underestimates $\bar{\chi}_{\Omega}$ at early times. These high initial values of the experimental $\bar{\chi}_{\Omega}$ (i.e., high mean concentration gradient) result from the blurring of the main bleaching line that occurs during the photobleaching procedure (see section 2.2). The fully saturated experiment $\left(S_{w}=1\right)$ is omitted since in that case the stretching has a minor effect on the concentration field.

Table 2. Model Parameters Used for the Investigated Saturation Degrees ${ }^{\mathrm{a}}$

\begin{tabular}{lcll}
$S_{\mathrm{w}}$ & $\mathrm{s}_{0}(\mathrm{~mm})$ & $D_{\text {eff }}\left(\mathrm{mm}^{2} \mathrm{~s}^{-1}\right)$ & $\overline{\nabla v}\left(\mathrm{~s}^{-1}\right)$ \\
\hline 0.83 & 20 & $1.40 \times 10^{-3}$ & 0.0092 \\
0.77 & 17 & $1.60 \times 10^{-3}$ & 0.0098 \\
0.71 & 25 & $1.90 \times 10^{-3}$ & 0.0106
\end{tabular}

${ }^{\mathrm{a}} S_{\mathrm{w}}$ : initial width of the line pulse $s_{0}$, effective diffusion coefficient $D_{\text {eff }}$ and mean velocity gradient $\overline{\nabla v}$.

\section{Discussion}

The presented Dirac injection experiments provide the first experimental evidence of the interplay between shear, compression, and diffusion at pore scale, under unsaturated conditions. The model of shear-enhanced mixing provides a framework to understand the evolution 
of the concentration gradient and scalar dissipation rate as a result of the evolution of the mean concentration and of the mixing scale (equation (7)). Stretching accelerates the decay of the mean concentration (equation (9)) and induces an initial compression of the mixing scale followed by a diffusive broadening (equation (11)). In the stretching regime, compression is stronger than diffusive growth, which implies that the mixing scale decays as $\bar{s} \sim t^{-1}$ (equation (11)) and that the average concentration in the tracer line $c_{m}$ remains approximately constant (equation (9)). This makes the mean concentration gradient increase as $\overline{\nabla c} \sim t$, and the scalar dissipation rate as $\bar{\chi}_{\Omega} \sim t^{2}$. At the mixing time $t_{\mathrm{s}}$, diffusive growth balances compressive effects due to stretching [Le Borgne et al., 2015], and $\overline{\nabla c}$ reaches a maximum. In the dissipation regime, the decay of concentration is enhanced by stretching, $c_{m} \sim t^{-3 / 2}$ (equation (9)), and the mixing scale grows diffusively, $\bar{s} \sim t^{1 / 2}$ (equation (11)). Therefore, the expected dissipation of gradients is $\overline{\nabla c} \sim t^{-2}$ and $\bar{\chi}_{\Omega} \sim t^{-4}$.

A decrease in $t_{\mathrm{s}}$ and a larger $\overline{\nabla c}\left(t_{s}\right)$ is observed as saturation decreases. This trend is well captured by equation (12). Indeed, a decrease in saturation $S_{w}$ implies an increase in the mean velocity and thus in the mean velocity gradient $\overline{\nabla v}$, which, according to equation (12), leads to a decrease in $t_{\mathrm{s}}$. However, for a small water content, the situation can be inverted, as shown by the plot obtained with a water saturation $S_{w}=0.71$. For that plot, $t_{\mathrm{s}}$ is the largest of the measured crossover times; in this case it is because $s_{0}$ is much larger than for the other two experiments: in equation (12) a larger $s_{0}$ results in a larger $t_{\mathrm{s}}$. The reason why the initial line width is much larger in that case is that the lensing-induced photobleaching, previously discussed in the Experimental protocol section, occurred to a much larger extent. On the other hand, the $\overline{\nabla c}\left(t_{s}\right)$ measured for $S_{w}=0.71$ lies in-between the values obtained for the two other saturations. This is explained by the fact that, due to the large initial width of the tracer line, an important amount of tracer is already in low velocity regions from the start of the experiment. This part of the tracer plume does not move advectively, it mainly diffuses; in the averaging of the concentration gradient over the entire system, such low velocity regions contribute low gradient values, thus compensating the important stretching that takes place at solute fingers in preferential flow paths.

Parameters used in the shear-enhanced mixing model are common in the soil physics community. A firstorder estimate of these parameters can be obtained from basic knowledge of the porous media (soil) properties. The bulk water saturation $\left(S_{w}\right)$ or water content $(\theta)$, effective diffusion coefficient (e.g., $D_{\text {eff }}=D \xi$, where $\xi$ is the saturation dependent tortuosity, Millington and Quirk [1961], Ghanbarian et al. [2013]), and mean velocity gradient $\overline{\nabla v}$ (note that $\bar{v}$ can be obtained as $q / \theta$, where $q$ is the Darcy velocity) can be inferred relatively easy. Of course the porous medium used in our experiments is a conceptualization of a much more complex reality. Besides the absence of gravity, parameters as porosity, pore size and connectivity are much larger than their common values in natural soils, while others, such as tortuosity, are smaller. Therefore, extrapolation of these results to natural soils should be taken with care.

These results obtained under Dirac injection can be compared to the mixing dynamics observed with a continuous solute injection [Jiménez-Martínez et al., 2015], indicate that in unsaturated conditions the scalar dissipation rate decreases significantly more slowly in time than under saturated conditions, even for a Péclet number smaller in the unsaturated experiments than in its saturated counterpart. This phenomenon was explained by the particular solute finger dynamics: longitudinal finger development, molecular diffusion mostly in the direction transverse to the mean flow, and hindering of transverse finger coalescence by the presence of air bubbles. Concentration gradients are sustained for a longer time as result of that dynamics as well as of the non-Fickian solute dispersion, which controls the concentration carried by fingers. In this work, we have shown that for a pulse line injection, two different regimes are obtained: an initial increase in the concentration gradients followed by a strong decay. The two types of experiments (with continuous injection and with pulse line injection) can be, in principle, related to each other since the derivative of an error function is a Gaussian distribution, so that the concentration field for a pulse line injection can be obtained, at any time, as the temporal derivative of the concentration field obtained at the same time with the corresponding continuous injection. In practice however, doing so results in a concentration field for the pulse line injection that is significantly noisy, since the derivative of the measured concentration field is much more sensitive to the experimental noise than the measured concentration field itself. Note however that although they are much more noisy, the time derivatives of the concentration fields measured for a continuous injection (data by Jiménez-Martínez et al. [2015], not shown here) show a behavior qualitatively similar to the results presented above, in terms of both dispersion $\left(\sigma_{x}\right)$ and mixing intensity (i.e., $\overline{\nabla c}$ and 
$\left.\bar{\chi}_{\Omega}\right)$. Hence, the experiments of [Jiménez-Martínez et al., 2015] and the present one are consistent with each other. This suggests that the sustained gradients observed by [Jiménez-Martínez et al., 2015] are also a consequence, beside the processes mentioned above, of the continuous source of solute mass.

The presented results and proposed model provide an understanding of transport and mixing in unsaturated porous media at spatial scales smaller than the size of the largest gas bubble (i.e., below the REV size in such systems), or, equivalently, at time scales smaller than the advective time necessary to travel over the size of the largest gas bubble. At much larger times, stretching is expected to have a minor impact on the concentration field, as diffusion becomes the dominant process, with eventually a change in the temporal behavior of $\overline{\nabla c}$ and $\bar{\chi}_{\Omega}$. How the scalar dissipation rate $\bar{\chi}_{\Omega}$ is expected to behave at these later times can be inferred from Equation (13). Indeed, at sufficiently large times, the first factor in this equation is proportional to $1 / t$ while the second factor $c_{0}^{2} /\left(1+4 \tau_{e}(t)\right)$ is equivalent to $c_{0}^{2} /\left(4 \tau_{e}(t)\right)$. The time behavior of the scalar dissipation rate is then controlled by that of the warped time $\tau_{e}(t)$, whose expression as a function of time is given by equation (10): at times for which the elongation experienced by the solute line can still be considered to be linear in time, $\tau_{e}$ grows as $t^{3}$, while at later times the stretching can be considered constant so that $\tau_{e}$ will vary as $t$. Consequently, at sufficiently large times one expects to see a regime for which $\bar{\chi}_{\Omega}$ will decrease as $t^{-4}$, followed by a transition to a regime for which it will vary as $t^{-2}$. None of these two regimes is really seen in Figure 7 due to the finite size of the experimental setup, but the approach towards the first of the regimes $\left(t^{-4}\right)$ is seen in the plots of the model (solid lines).

\section{Conclusions}

The experimental data set presented here sheds new light on the impact of water saturation on the dispersion and mixing dynamics following a pulse line injection. The developed photobleaching technique allows performing controlled Dirac injection experiments without disturbing the flow field and with a precision in the geometric definition of the solute line that is unprecedented in porous media flows. The subsequently measured high resolution pore scale concentration fields can be used to investigate (i) the dynamics of non-Fickian dispersion in unsaturated flows without the need of fitting a model to breakthrough curves, and (ii) the coupling between fluid stretching and diffusion that controls the evolution of concentration gradients, and therefore of mixing, at the pore scale. Although a similar analysis could in principle be done from data obtained by differentiating concentration fields measured under conditions of continuous injection, this would yield very noisy concentration fields.

During the experimentally available time scale, dispersion is found to shift from an approximate Fickian scaling in fully saturated conditions to a ballistic behavior in partially saturated conditions. This suggests that non-Fickian dispersion is determined by the existence of independent fast and slow channel with little diffusive exchange between them. Ballistic dispersion is expected to persist until the characteristic correlation time of the velocities carrying solutes across the medium. This characteristic time is thus found to increase significantly under unsaturated conditions due to the development of a highly heterogeneous velocity field, with coexistence of regions with high and very low velocities. After this ballistic regime, a slow transition to a Fickian dispersion regime is expected.

While molecular diffusion does not affect the ballistic dispersion behavior over the time of the experiments, it plays an important role in controlling the dynamics of mixing. While previous observations made under continuous injection conditions showed a slow monotonous decay of concentration gradients, two solute mixing regimes have been evidenced for conditions of Dirac injection. In the initial stretching regime, concentration gradients are enhanced by the compression induced by fluid deformation. This is followed by a dissipation regime during which diffusion overcomes compression, which results in a sharp decay of the concentration gradients. The transition between these two regimes occurs at a characteristic mixing time at which the mean concentration gradient is maximum. We have proposed a simple shear-enhanced mixing model that captures the mixing dynamics in unsaturated porous media under Dirac delta injection. It relates the maximum concentration gradient and the mixing time to basic characteristics of the unsaturated flow and associated solute transport, including the saturation degree, pore size, and effective pore scale diffusion. As saturation is lowered, the velocity heterogeneity, and thus the mean velocity gradient, is expected to increase. In the presented framework, this increase in shear induced deformation as saturation decreases implies a decrease in the mixing time and an increase in the maximum concentration gradient. This is 
consistent with our observations for the two largest saturations. However, below a water saturation threshold, the trend is inverted. This is explained by the fact that an important amount of tracer then resides in low velocity zones, in which the solute is transported mostly by molecular diffusing, which compensates the importance of stretching.

The considered experimental setup represents of course highly idealized geometry and conditions in comparison to the field, where the porosities are typically smaller $(\sim 0.3)$ and the tortuosities larger. Spatially heterogeneous wettability, film flow or displacement of the phases has not been considered here. Therefore, although we believe that the mixing mechanisms evidenced here are relevant at field scale, the extrapolation of the proposed model should be done considering the above mentioned limitations.

\section{Acknowledgments}

The authors gratefully acknowledge support from the EC2CO program of INSU/CNRS (project AO2014-906387). The experimental work was also supported by the Interreg project CLIMAWAT, EU-RDF INTERREG IVA France (Channel)-England program. J.J.-M. expresses his gratitude to Fondation Rennes I. Y.M. acknowledges support from RennesMétropole through an A.I.S. grant for equipment. The data used are available by contacting the corresponding authors.

\section{References}

Barbier, E. (2002), Geothermal energy technology and current status: An overview, Renew. Sust. Energ. Rev., 6, 3-65.

Benson, D., S. Wheatcraft, and M. Meerschaert (2000), The fractional-order governing equation of levy motion, Water Resour. Res., 36, 14131423.

Berkowitz, B., A. Cortis, M. Dentz, and H. Scher (2006), Modeling non-Fickian transport in geological formations as a continuous time random walk, Rev. Geophys., 44, RG2003, doi:10.1029/2005RG000178.

Birkholzer, J., and C. F. Tsang (1997), Solute channeling in unsaturated heterogeneous porous media, Water Resour. Res., 33, $2221-2238$.

Bolster, D., M. Barahona, M. Dentz, D. Fernandez-Garcia, X. Sanchez-Vila, P. Trinchero, C. Valhondo, and D. Tartakovsky (2009), Probabilistic risk analysis of groundwater remediation strategies, Water Resour. Res., 45, W06413, doi:10.1029/2008WR007551.

Bouchaud, J.-P., and A. Georges (1990), Anomalous diffusion in disordered media: Statistical mechanisms, models and physical applications, Phys. Rep., 195(4-5), 127-293.

Bouquain, J., Y. Méheust, and P. Davy (2011), Horizontal pre-asymptotic solute transport in a model fracture with significant density contrasts, J. Contam. Hydrol., 120-121, 184-197.

Bouwer, H. (2002), Artificial recharge of groundwater: Hydrogeology and engineering, Hydrol. J., 10, 121-142.

Brooks, R. H., and A. T. Corey (1964), Hydraulic Properties of Porous Media, Hydrol. Pap. 3, Colorado State Univ., Fort Collins, Colo.

Cuenca, A., and A. Bodiguel (2012), Fluorescence photobleaching to evaluate flow velocity and hydrodynamic dispersion in nanoslits, Lab Chip, 12, 1672-1679.

de Anna, P., J. Jimenez-Martinez, H. Tabuteau, R. Turuban, T. Le Borgne, M. Derrien, and Y. Méheust (2014a), Mixing and reaction kinetics in porous media: An experimental pore scale quantification, Environ. Sci. Technol., 48, 508-516.

de Anna, P., M. Dentz, A. Tartakovsky, and T. Le Borgne (2014b), The filamentary structure of mixing fronts and its control on reaction kinetics in porous media flows, Geophys. Res. Lett., 41, 4586-4593, doi:10.1002/2014GL060068.

de Barros, F., M. Dentz, J. Koch, and W. Nowak (2012), Flow topology and scalar mixing in spatially heterogeneous flow field, Geophys. Res. Lett., 39, L08404, doi:10.1029/2012GL051302.

de Dreuzy, J.-R., J. Carrera, M. Dentz, and T. Le Borgne (2012), Time evolution of mixing in heterogeneous porous media, Water Resour. Res., 48, W06511, doi:10.1029/2005WR004511.

de Gennes, P. G. (1983), Hydrodynamic dispersion in unsaturated porous media, J. Fluid Mech., 136, 189-200.

de Simoni, M., J. Carrera, X. Sanchez-Vila, and A. Guadagnini (2005), A procedure for the solution of multicomponent reactive transport problems, Water Resour. Res., 41, W11410, doi:10.1029/2005WR004,056.

Ghanbarian, B., A. G. Hunt, M. Sahimi, R. P. Ewing, and T. E. Skinner (2013), Percolation theory generates a physically based description of tortuosity in saturated and unsaturated porous media, Soil Sci. Soc. Am. J., 77(6), 1920-1929.

Gouet-Kaplan, M., and B. Berkowitz (2011), Measurements of interactions between resident and infiltrating water in a lattice micromodel, Vadose Zone J., 10(2), 624-633.

Haga, D., Y. Niibori, and T. Chida (1999), Hydrodynamic dispersion and mass transfer in unsaturated flow, Water Resour. Res., 35, 10651077.

Imamura, M., and M. Koizumi (1955), Irreversible photobleaching of the solution of fluorescent dyes. I. Kinetic studies on the primary process, Bull. Chem. Soc. Jpn., 28(2), 117-124.

Jha, B., L. Cueto-Felgueroso, and R. Juanes (2011), Fluid mixing from viscous fingering, Phys. Rev. Lett., 106, 194502.

Jiménez-Martínez, J., P. de Anna, H. Tabuteau, R. Turuban, T. Le Borgne, and Y. Méheust (2015), Pore scale mechanisms for the enhancement of mixing in unsaturated porous media and implications for chemical reactions, Geophys. Res. Lett., 42, 5316-5324, doi:10.1002/ 2015 GL064513.

Jiménez-Martínez, J., M. L. Porter, J. D. Hyman, J. W. Carey, and H. S. Viswanathan (2016), Mixing in a three-phase system: Enhanced production of oil-wet reservoirs by $\mathrm{CO}_{2}$ injection, Geophys. Res. Lett., 43, 196-205, doi:10.1002/2015GL066787.

Kapetas, L., I. Dror, and B. Berkowitz (2014), Evidence of preferential path formation and path memory effect during successive infiltration and drainage cycles in uniform sand columns, J. Contam. Hydrol., 165, 1-10.

Karadimitriou, N. K., V. Joekar-Niasar, M. Babaei, and C. A. Shore (2016), Critical role of the immobile zone in non-Fickian two-phase transport: A new paradigm, Environ. Sci. Technol., 50, 4384-4392.

Lahav, O., M. Kochva, and J. Tarchizky (2010), Potential drawbacks associated with agricultural irrigation with treated wastewaters from desalinated water origin and possible remedies, Water Sci. Technol., 61, 2451-2460.

Le Borgne, T., M. Dentz, and J. Carrera (2008), Lagrangian statistical model for transport in highly heterogeneous velocity fields, Phys. Rev. Lett., 101(9), 090,601.

Le Borgne, T., M. Dentz, D. Bolster, J. Carrera, J.-R. de Dreuzy, and P. Davy (2010), Non-Fickian mixing: Temporal evolution of the scalar dissipation rate in heterogeneous porous media, Adv. Water Resour., 33, 1468-1475.

Le Borgne, T., M. Dentz, P. Davy, D. Bolster, J. Carrera, J.-R. de Dreuzy, and O. Bour (2011), Persistence of incomplete mixing: A key to anomalous transport, Phys. Rev. E, 84, 015301.

Le Borgne, T., M. Dentz, and E. Villermaux (2013), Stretching, coalescence and mixing in porous media, Phys. Rev. Lett., 110, 204501.

Le Borgne, T., T. R. Ginn, and M. Dentz (2014), Impact of fluid deformation on mixing-induced chemical reactions in heterogeneous flows, Geophys. Res. Lett., 41, 7898-7906, doi:10.1002/2014GL062038. 
Le Borgne, T., M. Dentz, and E. Villermaux (2015), The lamellar description of mixing in porous media, J. Fluid Mech., 770, $458-498$. Lenormand, R. (1990), Liquids in porous media, J. Phys. Condens. Matter, 2(S), SA79.

Liu, Q., M. Donga, W. Zhoua, M. Ayub, Y. P. Zhang, and S. Huang (2004), Improved oil recovery by adsorptiondesorption in chemical flooding, J. Pet. Sci. Eng., 43, 75-86.

Løvoll, G., Y. Méheust, R. Toussaint, J. Schmittbuhl, and K. J. Måløy (2004), Growth activity during fingering in a porous Hele-Shaw cell, Phys. Rev. $E, 70,026301$.

Maraqa, M., R. Wallace, and T. Voice (1997), Effects of a degree of water saturation on dispersivity and immobile water in sandy soil columns, J. Contam. Hydrol., 25, 199-218.

Méheust, Y., G. Løvoll, K. J. Måløy, and J. Schmittbuhl (2002), Interface scaling in a two-dimensional porous medium under combined viscous, gravity, and capillary effects, Phys. Rev. E, 66, 051603.

Meunier, P., and E. Villermaux (2010), The diffusive strip method for scalar mixing in two dimensions, J. Fluid Mech., 662, 134-172.

Middleton, R. S., J. W. Carey, R. P. Currier, J. D. Hyman, Q. Kang, S. Karra, J. Jiménez-Martínez, M. L. Porter, and H. S. Viswanathan (2015), Shale and non-aqueous fracturing fluids: Opportunities and challenges for supercritical $\mathrm{CO}_{2}$, Appl. Energy, 147, 500-509.

Millington, R. J., and J. P. Quirk (1961), Permeability of porous solids, Trams. Faraday Soc., 57, 1200-1207.

Nutzmann, G., S. Maciejewski, and K. Joswig (2002), Estimation of water saturation dependence of dispersion in unsaturated porous media: Experiments and modelling analysis, J. Hydrol., 25, 565-576.

Orr, F. M. (2009), Onshore geologic storage of $\mathrm{CO}_{2}$, Science, 325(5948), 1656-1658, doi:10.1126/science.1175677.

Ottino, J. M. (1989), The Kinematics of Mixing: Stretching, Chaos, and Transport, Cambridge Univ. Press, Cambridge, U. K.

Padilla, I. Y., T. C. J. Yeh, and M. H. Conklin (1999), The effect of water content on solute transport in unsaturated porous media, Water Resour. Res., 35, 3303-3313.

Panfilov, M. (2010), Underground storage of hydrogen: In situ self-organization and methane generation, Trans. Porous Media, 85, 841-864.

Persson, M., S. Haridy, J. Olsson, and J. Wendt (2005), Solute transport dynamics by high-resolution dye tracer experiments-Image analysis and time moments, Vadose Zone J., 4, 856-865.

Pinela, J., S. Kruz, A. F. Miguel, A. H. Reis, and M. Aydin (2005), Permeability-porosity relationship assessment by 2-D numerical simulations, in Proceedings of the International Symposium on Transport Phenomena (ISTP-16), pp. 159-164, Pacific Center of Thermal-Fluids Engineering, Prague.

Pope, S. B. (2000), Turbulent Flows, Cambridge Univ. Press, Cambridge, U. K.

Ranz, W. E. (1979), Applications of a stretch model to mixing, diffusion, and reaction in laminar and turbulent flows, A/ChE J., 25(1), 41-47. Rhodes, M. E., and M. J. Blunt (2007), Advective transport in percolation clusters, Phys. Rev. E, 75(1), 011124

Rockstrom, J. E. A. (2009), A safe operating space for humanity, Nature, 461(7263), 472-475.

Rolle, M., C. Eberhardt, G. Chiogna, O. A. Cirpka, and P. Gradwohl (2009), Enhancement of dilution and transverse reactive mixing in porous media: Experiments and model-based interpretation, J. Hydrol., 110, 130-142.

Sahimi, M. (2012), Dispersion in porous media, continuous-time random walks, and percolation, Phys. Rev. E, 85, 016316.

Sani, A., M. Scholz, A. Babatunde, and Y. Wang (2013), Impact of water quality parameters on the clogging of vertical-flow constructed wetlands treating urban wastewater, Water Air Soil Poll., 224, 1488, doi:10.1007/s11270-013-1488-2.

Sebilo, M., B. Mayer, B. Nicolardot, G. Pinay, and A. Mariotti (2013), Long-term fate of nitrate fertilizer in agricultural soils, Proc. Natl. Acad. Sci. U. S. A., 110(45), 18,185-18,189, doi:10.1073/pnas.1305372110.

Sinton, D., D. Erickson, and D. Li (2003), Microbubble lensing-induced photobleaching ( $\mu$-blip) with application to microflow visualization, Exp. Fluids, 35, 178-187.

Stauffer, D., and A. Aharony (1994), Introduction to Percolation Theory, CRC Press, Baca Raton, Fla.

Tallakstad, K. T., H. A. Knudsen, T. Ramstad, G. Løvoll, K. J. Måløy, R. Toussaint, and E. G. Flekkøy (2009a), Steady-state two-phase flow in porous media: Statistics and transport properties, Phys. Rev. Letter, 102, 074502.

Tallakstad, K. T., H. A. Løvoll, G. Knudsen, T. Ramstad, E. G. Flekkøy, and K. J. Måløy (2009b), Steady-state, simultaneous two-phase flow in porous media: An experimental study, Phys. Rev. E, 80, 036308.

Toussaint, R., G. Løvoll, Y. Méheust, J. Schmittbuhl, and K. J. Måløy (2005), Influence of pore-scale disorder on viscous fingering during drainage, Europhys. Lett., 71(4), 583-589.

Toussaint, R., K. Måløy, Y. Méheust, G. Løvoll, M. Jankov, G. Schäfer, and J. Schmittbuhl (2012), Two-phase flow: Structure, upscaling, and consequences for macroscopic transport properties, Vadose Zone J., 11(3), doi:10.2136/vzj2011.0123.

Ursino, N., T. Gimmi, and H. Fluhler (2001), Dilution of non-reactive tracers in variably saturated sandy structures, Adv. Water Res., 24, 877885.

Vanderborght, J., and H. Vereecken (2007), Review of dispersivities for transport modeling in soils, Vadose Zone J., 6, $29-52$.

Vanderborght, J., et al. (2001), Overview of inert tracer experiments in key belgian soil types: Relation between transport and soil morphological an hydraulic properties, Water Resour. Res., 37, 2837-2888.

Villermaux, E. (2012), Mixing by porous media, C. R. Mec., 340(11-12), 933-943.

Werth, C. J., O. A. Cirpka, and P. Grathwohl (2006), Enhanced mixing and reaction through flow focusing in heterogeneous porous media, Water Resour. Res., 42, W12414, doi:10.1029/2005WR004511.

Williams, K. H., A. Kemna, M. J. Wilkins, J. Druhan, E. Arntzen, A. L. NGuessan, P. E. Long, S. S. Hubbard, and J. F. Banfield (2009), Geophysical monitoring of coupled microbial and geochemical processes during stimulated subsurface bioremediation, Environ. Sci. Technol., 43(17), 6717-6723.

Winograd, I. J. (1981), Radioactive waste disposal in thick unsaturated zones, Science, 212(4502), 1457-1464. 